\title{
Internet thinking is integrated into the practical study of smart home and interior design
}

\author{
Ying Deng
}

Jiangxi Vocational Techlege Of Industry \& Trade, Jiangxi, Nanchang, 330038
739960103@qq.com

\begin{abstract}
Under the impact of the Internet wave, many traditional industries have undergone great changes, people's way of life has also changed, humanization and automation is today's many people's demands on life, smart home also emerged, gradually become a mainstream trend in the market. Smart home interior design is a process that the development of home industry must go through, can effectively improve the quality of human life and living environment, bring people new interior design concepts and lifestyle. This paper studies the changes brought about by smart home to interior design, explores the limitations of smart home development, and analyzes the impact of smart home on interior design.
\end{abstract}

Keywords : Interior design, Smart home, internet

\section{互联网思维融入智能家居与室内设计的实践研究}

\section{邓蕅}

\author{
江西工业贸易职业技术学院 江西 南昌 330038 \\ 739960103@qq.com
}

\section{摘要}

在互联网浪潮的冲击之下, 许多传统行业发生了巨变, 人们的生活方式也随之发生变化, 人性化和自动化是现 今不少人对生活的要求, 智能家居也就应运而生, 逐渐成为市场中一种主流趋势。智能家居室内设计是家居行 业发展必然要经过的一个过程, 能够有效的提高人类生活品质和生活环境质量, 带给人们新的室内设计理念和 生活方式。本文就智能家居给室内设计带来的变化进行研究, 探索智能家居发展的局限性, 剖析智能家居给室 内设计带来的影响。

关键词: 室内设计; 智能家居; 互联网

\section{1. 我国智能家居产业现状}

\section{1. 智能家居的概念}

智能家居是很早以前就以提出的概念, 主要以室 内设计为主, 借用物联网、移动互联网和云计算等技 术, 让家庭生活环境实行智能化的管理, 重点强调的 是对家居物理环境的智能化改造。智能家居综合了系 统、服务、管理、结构、控制, 使用者通过网络实施 控制, 以达到改进生活环境, 使之变得更加舒适快捷。 互联网思维与家电产业融合在近几年来一直是行业 聚焦的重点, 室内设计也随着智能家居的发展而快速
改变着。

智能系统概念在 90 年代进入我国家具行业, 那 时的智能家居仅仅是一个概念, 但随着科技的不断发 展, 智能家居产品的落地已在国内如火如茶地展开, 特别是最近几年, 其发展速度远超先前业内的预期, 收到许多人的追捧。近几年小区智能化的完善和普及, 以及房地产行业的大爆, 都为智能家居行业带来了新 的机遇。理想的智能家居应该是能够优化人们的生活 方式, 为人们创造一个更加舒适、便捷、安全、健康、 环保的居住环境的。这样的智能家居大环境之下，室 内设计师应该给顾客设计一个既保留传统家居, 也能 使用智能化现代工具, 提供全方位的信息交换和远程 控制的环境。 


\section{2. 智能家居的分类}

智能家居的一大好处就是能够根据不同使用人 群提供对应的功能。智能家居以系统的方式在室内设 计行业中出现, 在互联网背景下, 智能家居系统包含 着安全防护系统、自动化系统、通信系统和文化系统, 不同的系统有不同的功能, 各个功能之间相互连接配 合。面对不同的人群智能家居能调整不同系统设置的 侧重点, 给使用者提供个性化的服务。智能家居一共 分为健康管理、能源管理、家庭安防几种, 不同的智 能家居类别对应着不同功能的强化。

健康管理类智能家居依托可穿戴设备, 使用多层 次智能感知采集信息, 对采集到的信息进行分析, 以 便人们随时监控自己的健康状况。在 2014 年, 海尔 公司就已推出海尔空气盒子, 这款产品可以实时监控 室内温度、湿度以及 PM2. 5、甲醛等有害物质, 并将 信息汇集整理分析, 报告给用户, 而用户可以通过这 些信息远程了解家中情况, 并对家中的空调和空气净 化器进行控制, 让用户在回家的时候就能及时享受到 良好的空气质量。许多公司针对用户的健康监控需求, 推出了智能手环产品, 用户可以随时监控自己的健康 状况, 对自己的健康水平了如指掌。现今业内还推出 了智能养老概念, 除了一些专门为老人设计的可穿戴 设备外, 还研发出了健康服务机器人, 为老年人提供 更加方便安全的健康服务。健康服务机器人利用多种 传感器, 通过对网络和其他智能家居的连接, 对居家 环境、人、事件进行全方面的感知和判断, 以达到对 老年人健康监测、生活起居等进行综合管理与服务的 目的。

能源管理类家居可以对家庭中水、电、气以及新 能源的使用进行实时的监测与控制。通过智能检测能 够了让用户了解自己的能源使用情况, 对家中能源管 理进行合理化管控, 减少对能源的浪费, 促成能源的 节约。这种智能家居体现了现代科技的优越性, 节能 环保的生活方式更是促进了社会的发展。比如智能插 座技术, 能够自动对室内用电情况进行检测和控制, 不仅控制了家庭用电成本, 还能保证室内用电安全。

家庭安防智能家居可以增强居家环境的安全性, 利用新型传感系统, 使用各类传感器、摄像机等安防 监测设备, 给用户住宅提供一个集感知、报警、紧急 求助为一体的综合性服务系统。传统的家庭安防设备 主要是防盗门和防盗网, 这样的防护效果一般, 对家 庭美观性也有影响。而智能安防家居能通过对应操作 提高安全防护级别。其中最具代表性和普遍性的就是 智能门锁, 智能门锁能实时监控家外情况, 及时给用 户做出提醒, 用户只能通过自己设置的密码打开门锁, 这样大大提高了家庭安全性。

\section{3. 智能家居现今发展情况}

智能家居的理念虽在业内十分火热, 但是智能家 居仍然有着一定的局限性, 存在着许多问题, 虽然智
能家居行业有着很大的商机，但想让智能家居成为人 们的生活方式, 还有很长的一段路需要走。

\section{3. 1. 行业标准的缺失}

在智能家居市场上, 许多智能家居品牌和产品都 没有标准可言。智能家居是个涉猎非常广的产业, 不 仅需要家居的设计, 还需要电子技术的利用。想要建 设智能家居产业, 必须设立一个统一行业标准, 让厂 家能够按照同样的标准来生产设备, 将不合格的企业 淘汰。很多设备由于使用的标准不同, 产品不能与其 他品牌互通, 给用户的使用带来巨大困难, 也对智能 家居行业有着极大影响。许多消费者在选购产品时都 期望能够购买到各个类型家居的顶尖水平产品, 而在 智能家居行业, 每个企业所发展的侧重点不同, 擅长 制造智能电视的公司并不一定能够生产出好的智能 冰箱, 于是消费者在想要购买几家公司的产品, 但又 被系统不互通问题阻碍，陷入了两难的局面。现阶段 智能家居行业要想发展, 必须解决这一问题, 给智能 家居产业带来全新的行业风气。

\section{3. 2. 系统复杂}

智能家居所需要的系统非常复杂, 需要融合各种 系统问题和模块问题, 将智能家居发挥最大价值, 而 这种情况也导致智能家居在安装过程中出现很多操 作复杂的问题。智能家居安装的门槛变高, 对于智能 家居产业也是一个急需解决的问题。而对于使用智能 家居的用户来讲, 不了解智能家居的系统和操作, 会 让原本希望变得更加快捷的生活变得更加复杂。许多 智能家居的操作页面显得十分复杂难懂, 并且智能家 居的技术并不能完全通过单纯的语音控制系统而进 行精准控制, 所以操作页面不能够复杂。智能家居生 产的目的就是带给使用者便捷快速的生活, 而无法满 足使用者的这一需求, 智能家居产业也就无法发展下 去。

\subsection{3. 成本较高}

智能家居是一个先进新兴的产业, 拥有着众多产 品功能, 也联合着众多产业, 需要借用国外的高科技 手段, 这也就致使智能家居的前期设计和制作的成本 较高。智能家居还需要根据不同用户的需求设计不同 的产品, 在安装过程中也需要经过调试和设置才能使 用, 这些都需要高昂的成本, 让最后的产品展现出了 高昂的价格, 使许多用户望而却步。智能家居既然要 发展, 就不能只把目标锁定在高收入的人群中, 只有 价格让广大消费者接受, 让大多数人都有能够购买产 品的能力, 智能家居才能成为真正以后人们生活的模 式。

\section{3. 4. 售后服务不理想}

现阶段智能家居的普及度较低, 购买智能家居不 
像传统家居这么方便快捷，销售方式一般是直接从厂 家订货，不仅购买麻烦，售后也不方便。厂家一般集 中于某几个地点, 但用户群体是遍布大江南北的, 当 智能家居出现问题时，也不方便进行售后服务，智能 家居的修理也比较麻烦。用户使用智能家居一段时间 后, 很可能因为种种麻烦, 最后废弃智能的生活方式, 最后依然使用传统家居。

\section{表 1：探索解决智能家居行业问题的办法}

\begin{tabular}{|c|c|}
\hline 智能家居存在的问题 & 对应措施 \\
\hline 行业标准不统一 & $\begin{array}{c}\text { 政府带头促进智能家居 } \\
\text { 行业统一标准, 让不符 } \\
\text { 合标准的企业进行产品 } \\
\text { 调整。 }\end{array}$ \\
\hline 系统复杂 & $\begin{array}{c}\text { 智能家居设计时应充分 } \\
\text { 考虑用户的使用感受, } \\
\text { 让智能家居的使用人性 } \\
\text { 化。 }\end{array}$ \\
\hline 成本较高 & $\begin{array}{c}\text { 智能家居行业聘请创新 } \\
\text { 性人才, 探索降低成本 } \\
\text { 方法, 自主带头创新, } \\
\text { 防止外国相传产业垄 } \\
\text { 断。 }\end{array}$ \\
\hline 售后较差 & $\begin{array}{l}\text { 智能家居企业增强售后 } \\
\text { 服务, 增加售后网点和 } \\
\text { 线上售后方式。 }\end{array}$ \\
\hline
\end{tabular}

\section{2. 智能家居产业发展方向}

\section{1. 让 “大家” 带动 “小家”}

智能家居的成本高昂, 在发展初期遇到了很多限 制, 但智能家居是未来人们生活方式的发展方向, 智 能家居产业可以与政府合作, 在学校、医院等地进行 示范安装, 让人们感受到智能家居的便利性, 吸引消 费者的注意力, 还能监测其运行效果。智能家居还可 以和房地产开发商进行合作, 在小区里安装智能设备, 提高小区的安全性，也能让智能设备走进人们的生活。 用户如果体验效果良好, 便会主动要求在自己家中安 装智能家居, 增加复杂的智能家居。通过这种方式, 能够吸引很多的用户开始使用智能家居, 最终使智能 家居广泛使用。

\section{2. 智能家居设计简单化}

智能家居现今在人们生活的普及程度上还处于 初步阶段, 许多人还不熟练智能设备的使用, 智能家 居的设计应该按照用户的实际需求设计, 一个简单方 便的智能家居可以给用户带来新奇的体验和良好的 使用感受, 也能带动用户使用智能家居的积极性。智 能家居应该按照用户的实际需求进行设计, 而不是只 将功能堆砌在一起, 给用户的使用造成困扰。智能家 居应该给用户提供一个愉悦的使用体验。在现今语音 识别系统仍然不能够十分精确, 所以智能家居也要对 操作页面进行设计, 使其条例清晰, 让消费者使用简
单易上手。

\section{3. 智能家居与能源结合}

智能家居是在电器设备的基础上建立的, 电器设 备要能够通过智能控制来实现操作, 在用电时更加智 能化操作。在城市用电高峰期, 智能家居通过调控电 器设备的电力, 在保证正常使用的基础上减少不必要 的能源消耗, 这样使电器设备的用电更加合理。智能 家居作为一个健康环保的设备, 应该支持新能源发电 设备, 发展太阳能、生物能、风能发电设备的应用。

\section{4. 智能电子商务}

传统的购物模式是人们去到商场和超市中挑选 商品, 将物品拎回家中, 在进入了互联网时代之后, 人们将购物的平台从商场转移到了小小的手机屏幕 之中, 电子商务得到了快速的发展, 让人足不出户也 能挑选商品, 享受服务。而将电子商务的平台转移到 智能家居中, 让购物的舞台变大。三星就曾推出过一 款智能冰箱, 在冰箱上添加了巨大的智能触控屏, 将 智能手机和冰箱相结合, 除了能对食品进行保鲜, 还 能利用食品清单, 监控家中其他智能设备, 阅读新闻, 通过冰箱的巨大智能触控屏就能实现下单, 购买需要 的食品, 它还具有语音控制功能, 让电子购物变得更 加简单快捷。

\section{3. 智能家居对室内设计的影响}

\section{1. 对室内风格的影响}

室内设计经过长时间的发展和创新, 形成了一些 具有代表性的审美风格。智能家居不仅仅影响了室内 设计风格, 也在于智能家居系统精心设计给人带来的 视觉印象有很大改变。智能家居都比较注重外观, 形 成了独具一格的审美形式，让产品有着强烈的现代感。 这样的设计风格也对室内设计师提出了要求, 需要室 内设计过程中考虑智能家居的风格特色, 让室内设计 风格与智能家居风格相呼应。

室内色彩也是室内风格重要的一环, 室内色彩的 改变可以大大影响人们对室内设计的视觉体验。色彩 在很大程度上决定了室内设计的风格特色。在室内色 彩设计中最根本的问题便是配色问题, 色彩效果取决 于不同颜色之间的相互关系，同一颜色在不同的颜色 背景条件之下，色彩的效果也会截然不同，这是色彩 所特有的敏感性和依存性，色彩之间的协调关系是室 内设计师需要重点关注的一环。智能家居系统是可以 提供色彩更换的，在用户进入室内后，系统可以根据 你的喜好调节色彩, 让用户在居住时能保持新鲜感, 让人感到舒适。

室内设计中灯光设置也十分重要。在传统家居设 计中, 灯光的调节需要手动调节, 而且灯光的色彩是 固定的，许多不人性化的灯光设置会让用户的使用体 
验变得糟糕，比如躺在床上才发现没有关灯，而灯光 控制的按钮离自己很远，这就给用户的生活带来了不 便。在智能家居中, 灯光设置是灵活多变的, 可以通 过智能设备或语音控制进行控制, 室内设计也可以基 于这一出发点进行设计, 尽可能的设置合理的自动控 制系统，设置时间性控制、区域性控制等各种模式， 实现自动按时、按区的关闭灯具, 但同时也预留了人 工干预的渠道, 这样便能适应消费者的各种需求, 营 造出不一样的室内环境系统, 灯光这一元素在室内设 计中也就不显得棘手，变得灵巧活泼起来。

\section{2 . 对室内陈设的影响}

智能家居还对室内家居的设计和摆放带来了影 响。在液晶、等离子电视机普及的现在, 电视机屏幕 尺寸越来越大, 争取给用户带来沉浸式的体验, 电视 的两边还会放置音响, 这也就要求室内设计时改变电 视柜的尺寸和高度。同时现在电脑桌成了许多家庭的 必要需求, 这也和互联网的发展有着极大关联。智能 家居也让家庭陈列饰品成为了过去式, 室内陈列作为 用户仅仅对美的需求的产品, 在日常生活中显得十分 不人性化, 陈列品在家中不易打理、容易损坏的特点 都让它们逐渐退出历史舞台。智能家居系统可以让这 些美感在屏幕之中体现, 比如盆栽、字画、雕塑、照 片都可以直接展示, 伴随着可以自由改变的灯光, 色 彩, 给室内设计带来了新鲜感、人性化。

\section{4. 室内设计如何打造智能家居环境}

住宅是人们生活的必需品, 影响着人们的身心健 康, 设计住宅也是在设计消费者的生活。智能家居系 统对室内设计的影响是巨大的。室内设计想要利用智 能家居系统给消费者打造一个良好的居住环境, 就要 将舒适性放在设计的第一位。消费者通常对智能家居 在家中的利用有着明确要求, 通常要求对家电能够实 现更加灵活方便的智能控制, 以最大化的功效将消费 者从家务劳动中解放出来。设计师要考虑到消费者回 到家中、待在家中、离开家的行动轨迹, 要让消费者 能够在生活中实现前一天晚上设定好家电的工作程 序与时间, 自己在外工作家中的家电也能够有着良好 的安排程序, 例如在消费者根据自己的日程设定好家 电工作后, 消费者回到家中就能感受到干净的室内环 境和舒适的室内温度, 在下雨天能够收好衣服, 关好 门窗等等。

设计师也需要考虑好住宅的安全性。智能家居的 安全防护和消防报警系统要被好好的利用起来, 在遭 遇火灾或地震等意外事件时, 智能家居的损坏不能够 影响住户的逃生, 同时智能家居也要在此类情况下实 现同步的报警，最大范围的保护住户安全。

同时也需要设计师秉持着绿色环保的设计理念, 运用移动设备和互联网通信, 营造出一个安全健康智 能家居环境应该是室内设计的初衷, 一个优秀的智能 家居环境，应该是经过精心的室内设计，给用户带来
人性化、个性化的使用体验。室内设计应该坚持以人 为本，将用户的需求放在第一位，根据用户的特征来 调整对智能家居的使用。智能家居的设置还需要拥有 美感, 将室内设计为一个和谐一体的环境, 将室内空 间设置成一个集科技、功能、艺术为一体的理想空间。 室内设计要充分考虑用户的使用体验, 让用户不需要 学习繁琐的智能家居操作, 不需要可以改变自己的生 活习惯, 也能体验到智能家居给生活带来的便利。

结论:

智能家居体现了现代生活的便利和环保, 让家居 中人性化得到了大幅的提高, 使人们提高了生活质量, 在生活的方方面面都能感受到科技的力量。智能家居 的发展也给室内设计带来了不小的冲击力, 室内设计 也应该随着时代的发展不断进步，用积极的心态接受 和利用智能家居, 利用创新的互联网思维将室内设计 与智能家居相融合, 为用户带来安全、舒适、便利的 生活环境。

\section{REFERENCES}

[1] Ke Shanbei. Multi-participation, breaking down barriers and promoting high-quality digital home services. Interpretation of Guiding Opinions on Accelerating the Development of Digital Home and Improving Living Quality [J]. China Construction, 2021(07):6-7.

[2] Guo Shilin. Design and implementation path of intelligent home control system based on embedded system. Electronic production, 2021(04) : $44-45+89$.

[3] Zhang Jizhu. Research and design of software for adjusting the Environment Comfort degree of intelligent family. Chongqing University of Posts and Telecommunications, 2020.

[4] Lulu. Advantages and disadvantages of intelligent design in residential space design. Modern Marketing (information edition), 2020(03) : 168.

[5] Wang Jun, Sun Zejun. Design and implementation of intelligent home monitoring and control system based on user behavior analysis [J]. Internet of Things Technology, 2020,10(09):71-74+79.

[6] Xiao Ding, Wang Ganyu, Cai Ming, Li Xiu. Research on implicit conflict detection method based on knowledge map in scene linkage of smart home $[\mathrm{J}]$. chinese journal of computers, 2019,42(06):1190-1204. 